\title{
Indicios arcaicos en la Colección Doncellas, Jujuy (República argentina). El «Yacimiento» 26 a la luz de un fechado radiocarbónico (4811 \pm 39 AP)
}

Vestiges archaïques de la Collection Doncellas, Jujuy (République argentine). «Le site » 26 à la lumière d'une datation au carbon 14 (4811ะ39 AP)

Archaic remains in the Doncellas Collection, Jujuy (Argentina). «Site» 26 in light of carbon dating ( $4811 \pm 39 \mathrm{BP})$

\section{Cecilia Pérez de Micou}

\section{OpenEdition}

Journals

Edición electrónica

URL: http://journals.openedition.org/bifea/2835

DOI: $10.4000 /$ bifea.2835

ISSN: 2076-5827

Editor

Institut Français d'Études Andines

Edición impresa

Fecha de publicación: 1 abril 2009

Paginación: 75-85

ISSN: 0303-7495

Referencia electrónica

Cecilia Pérez de Micou, «Indicios arcaicos en la Colección Doncellas, Jujuy (República argentina). El

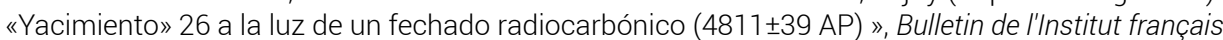
d'études andines [En línea], 38 (1) | 2009, Publicado el 01 octubre 2009, consultado el 17 noviembre 2020. URL : http://journals.openedition.org/bifea/2835 ; DOI : https://doi.org/10.4000/bifea.2835

\section{c)}

Les contenus du Bulletin de l'Institut français d'études andines sont mis à disposition selon les termes de la licence Creative Commons Attribution - Pas d'Utilisation Commerciale - Pas de Modification 4.0 International. 


\title{
Indicios arcaicos en la Colección Doncellas, Jujuy (República argentina). El "Yacimiento*» 26 a la luz de un fechado radiocarbónico (4811 \pm 39 AP)
}

\author{
Cecilia Pérez de $\mathrm{Micou}^{* *}$
}

\begin{abstract}
Resumen
En el presente trabajo se considera a la cestería, en sentido amplio, como una técnica textil que pone en relación dos o más elementos (trama y urdimbre) vegetales flexibles no hilados. Se analizan los materiales cesteros provenientes del enterratorio n. ${ }^{\circ} 26$ del sitio Doncellas, en la provincia de Jujuy, República argentina, adscrito a momentos tardíos de la ocupación prehispánica del noroeste argentino. Los resultados del estudio de la muestra completa de artefactos cesteros provenientes de todo el sitio indican una gran uniformidad técnico-morfológica en los mismos. La única excepción la constituye el enterratorio 26 en el que se dan artefactos cesteros de formas y técnicas que no aparecen en los demás conjuntos fúnebres. Un fechado radiocarbónico realizado sobre uno de ellos ubica este conjunto en el Período Arcaico Tardío, varios miles de años antes de lo esperado para la denominada cultura Doncellas, definida a partir de esta Colección.
\end{abstract}

Palabras clave: noroeste argentino, arqueología, Arcaico, cestería, AMS, funebria

\section{Vestiges archaïques de la Collection Doncellas, Jujuy (République argentine). "Le site » 26 à la lumière d'une datation au carbon 14 (4811 \pm 39 AP)}

\section{Résumé}

La vannerie est considérée comme une technique textile dont les éléments végétaux flexibles, non soumis à une procédure de tissage, sont reliés entre eux et créent des surfaces planes. Cet article

* En el Inventario de la Colección Doncellas se denominó «yacimiento» a cada uno de los enterratorios que constituían el cementerio de la localidad o sitio Doncellas.

** Investigadora CONICET/INAPL - Universidad de Buenos Aires. Tres de Febrero 1378 (1426), Buenos Aires, Argentina. E-mail: ccperez@arnet.com.ar 
analyse la vannerie de la tombe 26 du site Doncellas, province de Jujuy (République Argentine). Ce site correspond à l'époque récente de l'occupation préhispanique du nord-ouest argentin. Les résultats de l'étude réalisée sur la vannerie provenant du site indiquent qu'il s'agit d'un ensemble tout à fait homogène en ce qui concerne les formes et les techniques appliquées. Mais la tombe 26 constitue une exception. À la lumière de la datation absolue on peut dire qu'il s'agit d' un ensemble qui correspond à la période Archaïque récente, plusieurs milliers d’années avant l'apparition de la culture Doncellas, définie à partir de cette Collection.

Mots clés : nord-ouest argentin, archéologie, Archaïque, vannerie, AMS, traditions funêraires

\title{
Archaic remains in the Doncellas Collection, Jujuy (Argentina). «Site» 26 in light of carbon dating (4811 \pm 39 BP)
}

\begin{abstract}
In this text, basketry is considered in its broadest sense as a textile technique that relates two unspun flexible vegetal elements (weft and warp). We analyse two basketry objects found in burial site no. 26 of the Doncellas site, in the province of Jujuy, Argentina. These were dated as late in the pre-hispanic occupation of north-east Argentina. The results of the analysis of all of the samples of wickerwork taken from the entire site indicate uniformity in forms and techniques. The only exception to this is burial site 26, in which are found basketry artifacts of forms and techniques not found in the other funeral ensembles. A carbon dating on one of these placed the assemblage in site 26 in the Late Archaic Period, many thousands of years before that expected for the «Doncellas Culture», defined on the basis on this collection.
\end{abstract}

Key words: north-west Argentina, archeology, Archaic, basketry, AMS, burial traditions

La cestería arqueológica es una rica fuente de información acerca de las sociedades prehistóricas que las confeccionaron y/o usaron. Sus materias primas de origen biológico ofrecen la posibilidad de su datación absoluta, de la determinación de las especies vegetales usadas en tramas y urdimbres, posibilitando la localización de las zonas de recolección y la relación del contexto arqueológico con su ambiente (Pérez de Micou \& Ancibor, 1994; Péres de Micou, 2006). Al utilizar materias primas con escasa preparación previa, permiten el conocimiento casi directo de las técnicas de confección, ya que éstas son observables a ojo desnudo permitiendo la caracterización de los conjuntos cesteros de cada contexto arqueológico (Adovasio, 1977). Las formas finales, en caso de los ejemplares completos, podrían informar acerca de sus usos. Pueden dar cuenta de la esfera simbólica e ideacional de las sociedades que las produjeron, tanto al considerar su iconografía (Whiteford, 1988) como al integrar cada aspecto en un análisis totalizador. En este sentido, por tratarse de una manufactura todavía vigente en distintas sociedades, es pasible de ser objeto de trabajos etnoarqueológicos que generen ideas acerca de su significado social (Wendrich, 1999) en el pasado.

Sin embargo, como las evidencias de textiles prehistóricos se encuentran esporádicamente en el curso de las excavaciones arqueológicas, han tenido poco o ningún lugar en las consideraciones de los problemas arqueológicos de ciertas áreas (Miner, 1935-1936).

Este caso de estudio se refiere a los especímenes cesteros presentes en un enterratorio del sitio Doncellas del noroeste argentino, en cuyo análisis se ponen en juego algunos de los enfoques arriba citados. Los objetivos del mismo son: 
- Dar cuenta de las características especiales del enterratorio denominado Yacimiento 26, dentro de la uniformidad registrada en el resto de los enterratorios cuyo contenido forma la Colección Doncellas; y,

- explicar estas particularidades a partir de la evidencia aportada por la datación radiocarbónica de una de sus piezas.

\section{MATERIALES}

Los materiales analizados pertenecen a la Colección Doncellas, depositada desde mediados del siglo XX en dos museos de la Universidad de Buenos Aires (Museo Etnográfico en la ciudad de Buenos Aires y el Museo del Pucará en la localidad de Tilcara, Jujuy). Esta colección se formó a partir de campañas arqueológicas llevadas a cabo a comienzos de la década de 1940 en el poblado Doncellas, en la Puna de Jujuy (Casanova, 1938; 1943) y proviene totalmente de su cementerio. En él se abrieron alrededor de 200 enterratorios

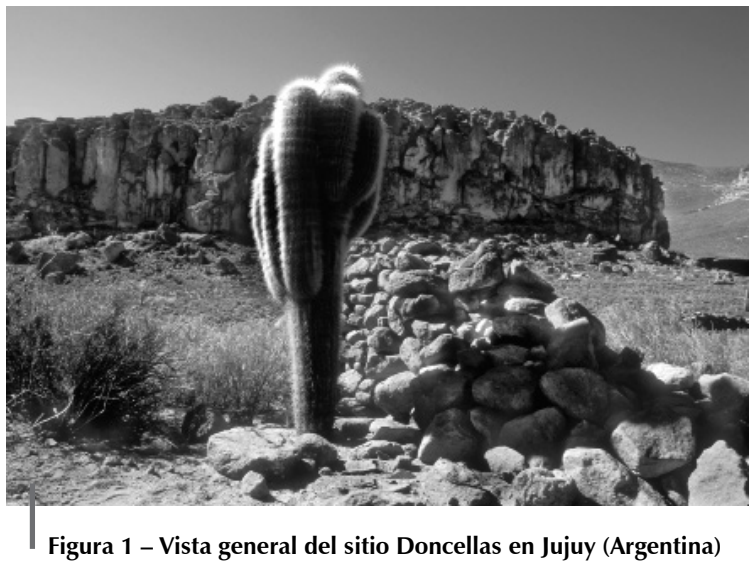
(yacimientos) alineados a lo largo de uno de los farallones (fig. 1) que enmarcan al sitio (Ottonello de García Reynoso, 1973). Similares en su arquitectura, presentaban paredes de piedra enlucidas de barro y pintadas, techo de cardón y pajas de la zona, y una puerta frontal. En la actualidad solo se conservan las paredes y las perforaciones en los farallones que indican el lugar de fijación de las vigas de los techos (fig. 2). Los fechados realizados sobre

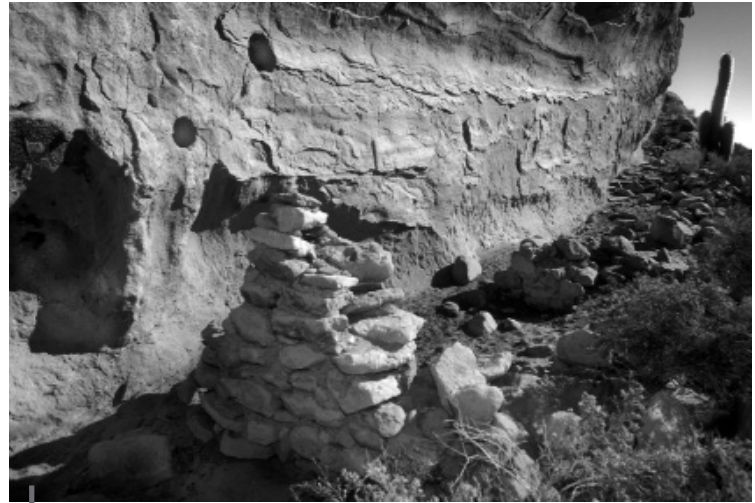

Figura 2 - Restos de un enterratorio. Se observan las perforaciones en que se fijaban las vigas de los techos cuerdas de la Colección Doncellas depositadas en el Museo Etnográfico dieron una edad de 700 años A. P. (Pérez de Micou, Ms, 1996). La fecha más antigua para el poblado Doncellas, excavado por Alfaro de Lanzone (1988), indica una antigüedad de 1000 años A. P.

Los materiales arqueológicos que integran la Colección muestran gran variedad de materias primas aplicadas en la confección de distintos artefactos: textiles de lana, maderas talladas, calabazas decoradas, cerámicas, cestería, cordelería, metalurgia. De todos ellos, se han analizados los tejidos (Rolandi de Perrot, 1979), las decoraciones de las calabazas (Hernández Llosas, 1983-1985) y la cestería (Pérez de Micou, 1996).

\section{1. La cestería de Doncellas}

La descripción de los ejemplares se basó en las técnicas de confección visibles en cada espécimen más que en las formas acabadas, ya que el material arqueológico se presentó en forma incompleta o decididamente fragmentaria, lo que impidió recuperar la forma original de cada pieza. 
El conjunto cestero, al igual que los demás materiales que integran la Colección Doncellas, presenta una escasa variabilidad. En el $87,50 \%$ de las cestas se utilizó la técnica espiral y solo el 6,25 \% de acordelado; otras técnicas cubren otro 6,30 \%. De las cestas en espiral (fig. 3), todas presentan como urdimbre (base) un haz de pastos y como trama (puntada) una puntada simple con variedades que no alcanzan a cambiar el aspecto general de la técnica de confección de las paredes. Esta persistencia se observa también en:

- El tipo de comienzos (centros). Se trata de centros normales cerrados (Adovasio, 1977).

- Las pocas terminaciones (bordes) que se han podido observar debido a su desgaste en todas las piezas, corresponden a bordes normales1.

- El ancho de las puntadas (entre 0,5 y $7 \mathrm{~mm}$ ).

- La separación entre urdimbres $(0 \mathrm{~mm})$ y la separación entre puntadas $(0 \mathrm{~mm})$.

- La escasez de ejemplares decorados (Pérez de Micou, 2001), todos realizados con la misma técnica de decoración estructural.

- Las materias primas utilizadas son locales. Se trata de gramíneas festucoideas y Cortaderia sp.
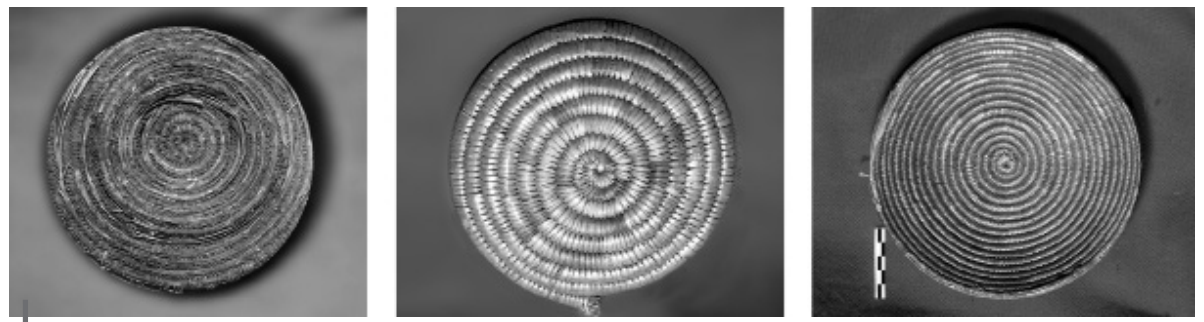

Figura 3 - Ejemplares de cestería espiral cerrado de puntada simple (Museo del Pucará) De izquierda a derecha: 42-1341 [1652]; 42-1387 [1658]; 42-2699 [1670])

Esta escasa variabilidad es aún más acentuada en la confección de los bozales (fig. 4) y las sogas, donde la torsión y la retorsión son las mismas (Z/S) en la totalidad de los casos. Por otra parte, solo hay dos tipos de especímenes de bozales: tipo A o complejos (88 \%)

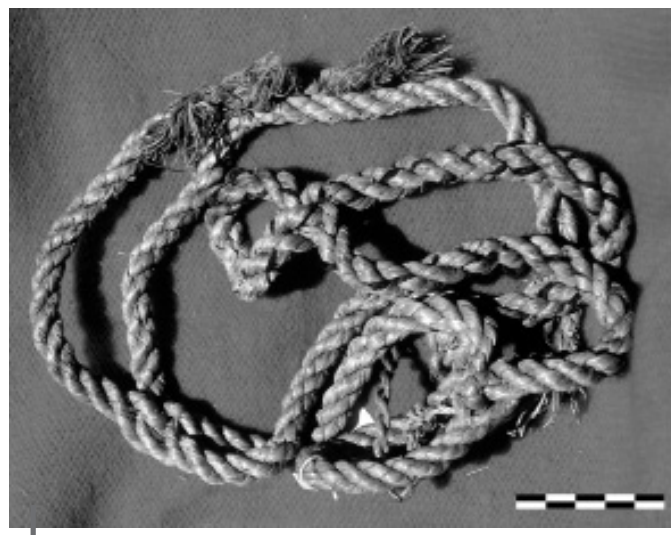

Figura 4 - Bozal tipo A

Museo Etnográfico: 42-623 caracterizados por la presencia de cinco uniones entre los diferentes ramales y tipo B o simples, que presentan solo tres uniones (12\%), con una dominancia absoluta del primer tipo sobre el total de los artefactos analizables (Pérez de Micou, 2001).

El conjunto de las denominadas «vinchas» (fig. 5) es algo más variable. Son artefactos con forma de circunferencia elaborados por torsión de haces de pastos (25\%), con técnica espiral $(13,60 \%)$ y técnica llana (61,40\%). Similares en su aspecto pudieron tener usos semejantes: las publicaciones citadas registran el hallazgo de restos humanos con esas «vinchas» en la cabeza tal como se exhibe en el Museo del Pucará, solo Alfaro de Lanzone da cuenta de su hallazgo en forma de trenza sin formar una circunferencia (Pérez de Micou, 2001).

1 El borde normal se realiza cosiendo el último espiral o coil con la misma puntada de todo el trabajo, reduciendo el grosor del mismo hasta hacerlo desaparecer. 


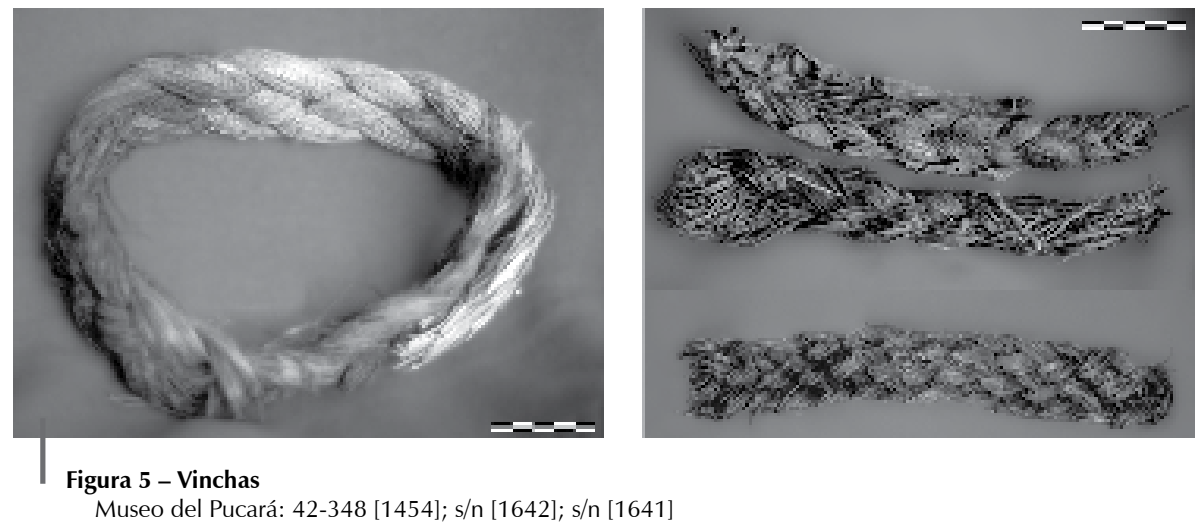

Esto permitió definir la cestería Doncellas como poco variable técnicamente y sin decoración, sumándola a lo que se llamó históricamente en la Arqueología argentina el Puna Complex², un conjunto artefactual perteneciente a los pastores de altura, definido así por Bennet (1948) y posteriormente por Lafón y Casanova, quienes hablan de una Cultura Atacameña con rasgos locales, denominada Cultura Atacameña de tipo Doncellas (Casanova, 1943; Lafón, 1965). Esta escasa variabilidad se constata también en los otros materiales analizados (calabazas pirograbadas y el tejido) y en los observados por nosotros durante la revisión de la Colección (palas de piedra y palas de madera, por ejemplo). Es coherente, también, con la repetición del planteo constructivo de los enterratorios del sitio.

\section{LA CESTERÍA DEL YACIMIENTO 26}

Sin embargo, como toda regla, la de Doncellas tenía su excepción. Se trata del Yacimiento 26 en su conjunto. Este enterratorio no contiene vestigios cerámicos, ni metalúrgicos o relacionados con la tecnología textil andina. También están ausentes en él, los artefactos pirograbados sobre calabazas, las palas de piedra, las sogas y bozales, que son los más representados en el resto de los enterratorios registrados. Contiene, en cambio, además de tecnofacturas sobre materias primas vegetales, partes humanas momificadas, un punzón y una punta de hueso. Esta información proviene en su totalidad del Libro de Inventario del Museo Etnográfico3 ${ }^{3}$ único documento público conservado sobre la Colección Doncellas.

Más específicamente, al analizar los materiales cesteros, no se halló ninguno que cumpliera con las características descritas más arriba para la cestería Doncellas. Se trata de un grupo de

2 El Puna Complex se componía de «... implementos de tejer (ovillos, torteros, husos, etc.), bolsas, cestos, trenzas de paja, fragmentos de cuero y tejidos de lana, arcos, flechas, vasitos de cerámica tosca, 〈hebillas», cuchillones de madera, es decir, los elementos ergológicos típicos de una cultura como la puneña, de pastores y agricultores con especialización en textiles y una cerámica tosca, técnicamente poco elaborada» (Alfaro de Lanzone, 1988: 104).

3 Yacimiento 26. Inventario del Museo Etnográfico de la Facultad de Filosofía y Letras de la UBA:

- 42-1113: Punta de flecha de hueso.

- 42-1114: Punzón-espátula.

- 42-1115: Dedo momificado.

- 42-1116: Sección de hueso quemado.

- 42-1117: Diadema de lana con aplicaciones de concha, se desglosaron dos; una de las diademas fue al Museo del Pucará.

- 42-1118: Objeto de lana con aplicaciones de concha.

- 42-1119: Cuero labrado (roto). 
piezas que se apartan del conjunto y están confeccionadas con técnica acordelada (twined) o, mediante las denominadas por Adovasio (1977), misceláneas. En un trabajo anterior (Pérez de Micou, 2002) se presentó la descripción de los cuatro artefactos cesteros con el objetivo de marcar las diferencias entre estos y los estudiados en la Colección completa. En este trabajo se focalizará en los realizados en técnica acordelada (twined) ya que varios autores han sugerido su uso anterior a la técnica espiral, en algunos sitios arqueológicos americanos (cf. Connolly et al., 1995) y la evidencia lograda hasta ahora parece confirmar esa presunción.

En Perú, la cueva Guitarrero presenta esta técnica en componentes anteriores a la aparición de cultígenos (Adovasio \& Lynch, 1973). En el sudoeste norteamericano —una zona caracterizada en los momentos tardíos del poblamiento indígena por la confección de cestería en espiral - la técnica más antigua, recuperada en Cueva Daisy, California, está confeccionada con técnica acordelada y retrotrae la aparición del tejido en la región unos 4000 años.

Asimismo, el noroeste argentino cuenta con algunos sitios arqueológicos que dan cuenta de la antigüedad de esta técnica. En Inca Cueva 4, por ejemplo, estudiada en la década de 1970, se encontraron fragmentos de cestería acordelada simple cerrada en el fondo de uno de los pozones excavados en el interior del espacio de habitación definido dentro de la cueva (Aschero, 1979). Recientes fechados le otorgan una antigüedad de $5146 \pm 41$ años A. P. (AA65137).

Por esto, nos interesó particularmente el estudio de los artefactos confeccionados en técnica acordelada de este enterratorio, asociado a materiales totalmente separados del Puna Complex. Manejábamos la hipótesis de su adscripción a un período anterior del poblamiento de la Puna que el indicado por los fechados conocidos para Doncellas.

\section{1. Cestería acordelada en el Yacimiento 26}

Se trata de dos ejemplares muy distintos entre sí. El primero es el «pendiente» (42-1118 [1654])4, posible fragmento de honda, de forma suboval, decorado con cuentas enhebradas en una de sus caras (fig. 6). La técnica general de confección es el acordelado cerrado simple con inclinación de tramas en S. Las urdimbres están formadas
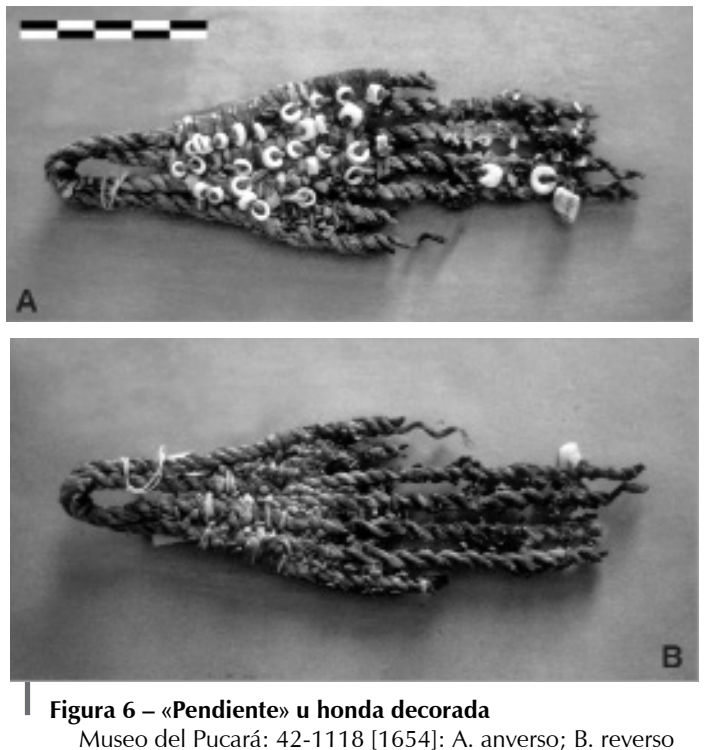

-42-1120: Diadema de plumas.

- 42-1121: Parte de un tejido de esparto.

- 42-1123: Cabeza momificada. En la trenza lleva arrollada una sarta de cuentas.

- 42-1124: Fragmento de tejido de lana y cuero (lote).

No se conoce la ubicación exacta de este enterratorio dentro del paraje Doncellas ni las características arquitectónicas del mismo. Se carece de esa información para todos los «yacimientos» del sitio.

4 Los números son los correspondientes al inventario del Museo Etnográfico de Buenos Aires y el número entre corchetes [ ] es el que corresponde a la misma pieza en el Museo del Pucará en Tilcara. 
por cordeles Z/ss de 3,6 a 4,5 mm de espesor. Tiene 2 urdimbres por $\mathrm{cm}$. Las 2 urdimbres que forman los orillos son cordeles Z/sss. Con respecto a la materia prima de las urdimbres no se pudo determinar su género ya que no fue posible extraer muestras que conservaran caracteres diagnósticos (Elena Ancibor, comunicación personal).

Las tramas son tientos de 3,2 a 4,3 mm, con 3 a 4 tramas por $\mathrm{cm}$.

La confección se realizó utilizando el cordel del orillo como marco. Paralelas a los dos orillos se tendieron 5 urdimbres y las tramas trabajaron perpendicularmente del modo que define la técnica de acordelado. El extremo que se conserva es un ojal formado por el doblez del orillo. El otro extremo está deshecho. La similitud entre esta descripción y la realizada por Rolandi de Perrot (1979) para las hondas de lana de la Colección Doncellas nos llevó a cambiar su denominación de «pendiente» por la de «honda»:

«El procedimiento de confección es bastante similar en todas. Consiste generalmente en un cuerda de tres cabos, cada cabo formado por varios elementos. A esta cuerda, cuando llega a la parte central de la honda, se le separan los 3 cabos, que van a actuar ahora como hilos de urdimbre, y se pasa la trama por arriba y por abajo de cada hilo de urdimbre. Cuando realizan la abertura separan dos cabos para un lado y uno para el otro, agregándole a este último otro elemento, y siguen pasando la trama. Concluida la parte central, en el otro extremo reúnen todos los elementos que actuaron de urdimbre y el que actuó de trama, y forman la soga. Otras veces se forma la honda doblando algunos elementos en la parte central, los que posteriormente constituirán la soga en una de las terminaciones, esto permite la formación de un asa por donde va a pasar la soga del otro extremo» (Rolandi de Perrot, 1979: 23).

El espécimen estudiado por nosotros no está confeccionado con lanas, es más grande $(150 \mathrm{~mm}$ de alto [fragmentado], por $49 \mathrm{~mm}$ de ancho) y está decorado en una faz. Sin embargo, la similitud de su confección con la de las hondas descritas en el tejido de la misma Colección coloca en esa categoría al «pendiente» de Doncellas; su decoración, en una sola de sus caras, avalaría este uso. En efecto, la decoración se realizó mediante el enhebrado, sin orden aparente, de cuentas rosadas (solo una negra) superpuestas y cosidas a la estructura textil. Es un trabajo posterior a la confección de la pieza y, hasta posiblemente, posterior a su fragmentación ya que los elementos de la decoración se encuentran completos. Este es el único ejemplar de la colección en que la decoración sería post confección del mismo.

El segundo ejemplar es el denominado Fragmento de tejidos de fibras vegetales (42-1117 [1664]).

Se trata de dos fragmentos rectangulares (530/550 por 185/280 mm) confeccionados con técnica acordelada cerrado simple con inclinación de trama en $\mathrm{S}$ (fig. 7). La urdimbre es un cordel vegetal Z/ss de $2 \mathrm{~mm}$ de espesor y torsión fuerte

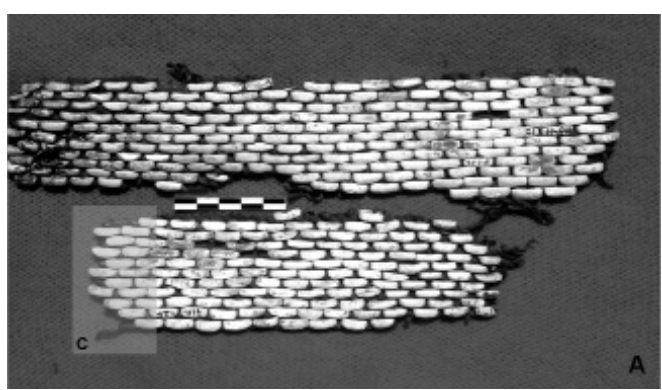
(5 torsiones/cm). Hay 2 urdimbres/cm.

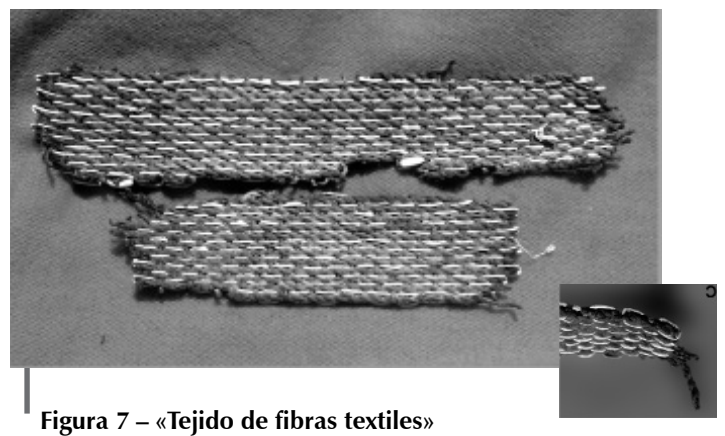

Figura 7 - «Tejido de fibras textiles»

Museo del Pucará: 42-1117 [1664]: A. anverso; B. reverso; C. detalle del borde 
La trama es un cordel vegetal S/zz de 4-5 mm de espesor, hay una separación entre tramas de 1,7 a $3 \mathrm{~mm}$ y aparecen 3 tramas/cm. En este caso tampoco pudo determinarse el género de la planta utilizada.

No presenta bordes, orillos ni comienzo del trabajo. Las urdimbres parecen cortadas al ras. Una de las caras del tejido está cubierta totalmente, por la parte plana, de cuentas blancas de material posiblemente óseo o malacológico que han sido enhebradas durante la confección del espécimen y que dan un aspecto de superficie eslabonada o articulada. Estas cuentas han sido enhebradas en las urdimbres y se colocan en forma alternada.

Hemos podido observar un ejemplar similar a éste entre las fotografías que nos facilitara Rolandi de Perrot de los materiales de la Colección privada del Dr. Torres Aparicio pertenecientes al sitio Inca Cueva 4 (Jujuy, Argentina). Estos materiales provienen de una recolección, realizada en 1936, de ocho inhumaciones de adultos y niños en la superficie de la cueva 4 de Inca Cueva. Según Aschero (1979) estos hallazgos, en la que denominara capa $1 \mathrm{~b}$, guardan relación con los realizados por él en la capa 2 que fuera posteriormente fechada entre 10620 y 9230 A. P. (García, 1997).

\section{DISCUSIÓN}

Una de las hipótesis que se tuvo en cuenta fue que se tratara de un enterratorio mucho más antiguo que los demás del sitio Doncellas (cuya edad ronda los 700 años A. P. hasta el contacto hispano-indígena) (Pérez de Micou, 1996). La inexistencia de metales y de cerámica harían suponer un contexto arcaico y esto estaría avalado por la datación relativa del material de Inca Cueva, cueva 4, perteneciente a la Colección del Dr. Torres Aparicio y que guarda similitud con el aquí descrito en segundo lugar como «tejidos de fibras textiles».

Con respecto a las técnicas, la presencia del acordelado, inexistente en los otros artefactos cesteros analizados en la Colección, podría indicar una gran profundidad temporal. Su uso está documentado a lo largo de toda la secuencia de ocupación en el noroeste argentino y en Perú. Pero, si bien es cierto que los fechados más antiguos, hasta ahora indican que la técnica de acordelado (twined) en sus distintas variantes (simple, diagonal y envolvente) está asociada a ellos tanto en América del Norte (California) como en el noroeste argentino, también hay que aclarar que esas técnicas no desaparecen posteriormente de los registros arqueológicos, y perduran en las tecnofacturas cesteras actuales, lo cual no las habilita como un indicador cronológico relativo.

Con respecto a la forma de los materiales, también se apartan de la generalidad ya que ésta mostraba cestos, en su mayoría pequeños, cóncavos o subcóncavos pero con una casi obvia función de contenedores. En el «yacimiento» 26, en cambio, ninguno de los objetos está asociado a esa función, por el contrario, parecerían objetos de uso personal, quizás vestidos o adornos corporales.

Habíamos definido a la cestería de Doncellas como no decorada ya que esa característica se confirmó en la mayoría de los artefactos analizados. Este conjunto, en cambio, presenta aplicación de cuentas en el denominado «pendiente» (honda). Esta no fue reparada, sino decorada muy probablemente para su uso como ofrenda (reciclaje). En el «tejido de fibras textiles» la decoración es estructural lo que implica una decisión previa a la confección, como es la inclusión de cuentas en las urdimbres. 


\section{1. Fechado de un artefacto del Yacimiento 26}

Ofrendas diferentes que implican prácticas fúnebres diferentes ofrecidas a un personaje destacado del resto de la comunidad y contemporáneo con ella. O relicto de las prácticas fúnebres de una sociedad más antigua, cuya ubicación en el tiempo deba relacionarse con los hallazgos de la capa 1 b de Inca Cueva 4. El envío de una muestra del ejemplar 42-1117 [1664] al Laboratorio de Física de la Universidad de Arizona, aportó la información que necesitamos para contrastar estas ideas. Se trata de un fragmento de cordel vegetal extraído de una de las urdimbres del ejemplar, que arrojó el siguiente resultado:

\begin{tabular}{|c|c|c|c|}
\hline AA65130 & DC13 & Fmodem & C 14 age BP \\
\hline & $-24,9$ & $0,5494 \pm 0,0027$ & $4811 \pm 39$ \\
\hline
\end{tabular}

Esta edad aproxima cronológicamente al Yacimiento 26 de la Colección Doncellas a sitios adjudicados al Arcaico Tardío en la Puna argentina. Por ejemplo: el sitio Inca Cueva 7 — Cuya edad es apenas unos 600 años más recientes - presenta una tecnología textil totalmente separada de la que presentan los sitios pertenecientes al Puna Complex y, por el contrario, muy cercana a la aquí descrita para el Yacimiento 26, como la honda publicada por Aguerre et al. (1973).

Además, como ya se dijo, un artefacto similar al datado para este trabajo fue exhumado en la superficie del sitio Inca Cueva 4 en asociación a enterratorios, de modo que su edad se había calculado, en forma relativa, como posterior a los 5000 años A. P.

\section{CONSIDERACIONES FINALES}

Parece claro, entonces, adjudicar a este enterratorio, no ya a un episodio especial de las comunidades pastoras puneñas inmediatamente anteriores al contacto incaico, sino como pertenecientes al Período Arcaico Tardío, habida cuenta de este fechado absoluto y del conjunto de evidencias artefactuales, tomadas como parte de lo que Aschero (1994) Ilama «paquete de rasgos diagnósticos», aplicable a la comparación de grandes áreas en amplios niveles de síntesis. Su idea de tomar al período arcaico como un proceso durante el cual la memoria acumula experiencias producidas en la interacción tecnología-ambiente que derivará en la experimentación agrícola y ganadera, permite ver estas tecnologías textiles sobre materias vegetales como parte de esa experimentación, si bien no conducente a cambiar la dieta, sino al dominio de estas manufacturas, que, a juzgar por la complejidad de las aquí descritas parecen contar con un largo proceso de transmisión.

Creemos importante el estudio de este enterratorio 26 ya que aporta información sobre las costumbres funerarias de ese momento, poco conocidas en la parte argentina de la región puneña y abre la posibilidad de investigar el paraje Doncellas cuya ocupación arcaica no había sido sugerida hasta ahora.

La cultura Doncellas, definida por Lafón (1965) a partir de la colección realizada por Casanova y confirmada por estudios posteriores (Alfaro de Lanzone, 1988), selló las posibilidades de marcar diversidades dentro del sitio del mismo nombre, siendo atribuido en su conjunto a los momentos inmediatamente anteriores a la expansión inca en el noroeste argentino y la posterior entrada del europeo. Sin embargo, este fechado indica que Doncellas fue ocupado $\mathrm{o}$, al menos, utilizado como lugar de entierro, desde varios milenios antes de lo que se suponía. En este sentido, cobra interés la cueva Tajuera, trabajada por Alfaro de Lanzone, 
en las cercanías del poblado de Doncellas. En ella hace ya muchos años describimos algunos artefactos perecederos - un carcaj de cardón, revestido de cuero cosido, un fragmento de cestería realizado en técnica de acordelado abierto (Open twined) — que se diferenciaban completamente de los materiales recuperados en la excavación del poblado de Doncellas. Lamentablemente, las técnicas aplicadas a la excavación de este sitio no permitieron definir niveles de ocupación, considerándose al total del contexto excavado como perteneciente a un solo componente. Por lo que pudimos observar en una prospección en 1995 y, según los pobladores que participaron del trabajo de campo, no se dejaron testigos para realizar excavaciones posteriores. De modo que sería necesario volver con otra mirada al paraje Doncellas, su poblado y cementerio prehispánico así como también continuar la labor empezada en el estudio de la colección a fin de redefinir su contenido.

La cestería como el resto de los textiles, al usar materias primas orgánicas, se constituye en fuente de primer nivel a la hora de establecer cronologías absolutas, especialmente con el desarrollo de las técnicas de datación por aceleración de partículas (AMS) que se aplican a cantidades ínfimas de material respetando la integridad de los fondos museísticos. Dedicar fondos al fechado de los materiales orgánicos de esta y otras colecciones arqueológicas similares sumará información certera sobre sitios, especialmente aquellos cuya excavación actual es imposible por su estado de preservación.

\section{Agradecimientos}

Esta investigación pudo comenzarse en 1985 gracias al apoyo del Instituto Nacional de Antropología y del Museo de Tilcara de la Universidad de Buenos Aires. Se completó gracias a subsidios otorgados a la autora por la Agencia Nacional de Promoción Científica (1998), la Secretaría de Ciencia y Técnica de la UBA (UBACYT 1996-2000) y CONICET (PIP 2467) y el Laboratorio de Física de la Universidad de Arizona.

A Barth Bagley por la realización del Abstract.

A Luis, Paula y Valeria Micou quienes colaboraron en la edición del presente trabajo.

A los evaluadores.

\section{Referencias citadas}

ADOVASIO, J., 1977 - Basketry technology. A guide for identification and analysis, 182 pp.; Chicago: Aldine Publications Co.

ADOVASIO, J. \& LYNCH, T., 1973 - Preceramic textiles and cordages from Guitarrero Cave, Perú. American Antiquity, 38 (1): 84-90.

AGUERRE, A. M., ASCHERO, C. \& FERNÁNDEZ DISTEL, A., 1973 - Hallazgo de un sitio acerámico en la Quebrada de Inca Cueva (Provincia de Jujuy). Relaciones, VII: 197236; Buenos Aires: Sociedad Argentina de Antropología.

ALFARO DE LANZONE, L., 1988 - Investigación en la Cuenca del Río Doncellas. Dpto. de Cochinoca, Provincia de Jujuy. Reconstrucción de una Cultura olvidada en la Puna Juneña, Jujuy: Gobierno de la Provincia de Jujuy.

ASCHERO, C. A., 1979 - Un asentamiento acerámico en la Quebrada de Inca Cueva (Jujuy). Informe preliminar sobre el sitio Inca Cueva cueva 4. Actas I Jornadas de Arqueología del noroeste argentino. Antiquitas, 2: 159-183.

ASCHERO, C. A., 1994 - Reflexiones desde el Arcaico tardío (6000-3000 AP). Rumitacana, Revista de Antropología. Dirección de Antropología de Catamarca, 1 (1): 13-17. 
BENNET, W., 1948 - Northwestern Argentine Archaeology; New Haven. Yale University Publications in Anthropology 38.

CASANOVA, E., 1938 - Excursión arqueológica a la Puna de Jujuy. Revista de Geografía Americana, 5 (57): 381-393.

CASANOVA, E., 1943 - Comunicación acerca del Yacimiento de Doncellas. Boletín de la Sociedad Argentina de Antropología, 5-6: 80-81.

CONNOLY, T., ERLANDSON, J. \& NORRIS, S., 1995 - Cestería y cordelería del Holoceno Temprano de la Cueva «Daisy» isla San Miguel, California. American Antiquity, 60 (2): 309-318.

GARCÍA, L. C., 1997 - Inca Cueva: ocupación a partir del Formativo Inferior Inicial. Avances en Arqueología, 3: 71-75; Buenos Aires: Universidad de Buenos Aires, Facultad de Filosofía y Letras.

HERNÁNDEZ LLOSAS, M. I., 1983-1985 - Las calabazas prehispánicas de la Puna Centro meridional (Jujuy, Argentina). Análisis de sus representaciones. Anales de Arqueología y Etnología, 38 (40): 77-158; Universidad Nacional de Cuyo.

LAFÓN, C. R., 1965 - Tiempo y cultura en la provincia de Jujuy. Etnia, Museo Etnográfico Dámaso Arce, 2: 1-19.

MINER, H., 1935-1936 - The Importance of Textiles in the Archaeology of the Eastern United States. American Antiquity, 1: 181-192. A Quaterly Review of American Archaeology. Published by The Society for American Archaeology.

OTTONELLO DE GARCÍA REYNOSO, M., 1973 - Instalación, Economía y cambio cultural en el sitio tardío de Agua Caliente de Rachaite. Publicaciones, Dirección de Antropología e Historia, 1: 23-68.

PÉREZ DE MICOU, C., 1996 - Los artefactos sobre materias primas vegetales flexibles de la colección Doncellas del Museo Etnográfico (Buenos Aires) y el Museo del Pucará (Tilcara). Tesis para optar al grado de Doctor de la Universidad de Buenos Aires. Ms.

PÉREZ DE MICOU, C., 2001 - Tecnología cestera en la Colección Doncellas (Jujuy, Argentina). Boletín Museo de Arqueología y Antropología, 4 (1): 20-26.

PÉREZ DE MICOU, C., 2002 - Cestería en contextos arqueológicos fúnebres del noroeste argentino. In: Actas II Jornadas internacionales sobre textiles precolombinos (V. Solanilla Demestre, ed.): 333-346; Universitat Autónoma de Barcelona, Department d’Art. Institut Catalá de Coperació Iberoamericana.

PÉREZ DE MICOU, C., 2006 - Cuerdas, cestas, esteras... Variaciones sobre la tecnología cestera. In: El modo de hacer las cosas. Artefactos y ecofactos en arqueología (C. Pérez de Micou, ed.): 171-186; Buenos Aires: Universidad de Buenos Aires, Facultad de Filosofía y Letras, Departamento de Ciencias Antropológicas.

PÉREZ DE MICOU, C. \& ANCIBOR, E., 1994 - Manufactura cestera en sitios arqueológicos de Antofagasta de la Sierra, Catamarca. Journal de la Société des Américanistes, 80: 207-216.

ROLANDI DE PERROT, D., 1979 - Los tejidos del río Doncellas, provincia de Jujuy. Antiquitas. In: Actas Jornadas de Arqueología del noroeste argentino: 22-73; Facultad de Filosofía y Letras, Universidad del Salvador.

WENDRICH, W., 1999-Theworld according to basketry. An ethno-archaeological interpretation of basketry production in Egypt, 492 pp.; Leiden: Research School of Asian, African and Amerindian Studies (CNWS), Universiteit Leiden, The Netherlands.

WHITEFORD, A. H., 1988 - Southwestern indian baskets. Their history and their makers, 219 рр.; Santa Fe, Nuevo México: School of American Research Press. 